\title{
Advances in microalgal biomass/bioenergy production with agricultural by-products: Analysis with various growth rate models
}

\author{
Hee-Jeong Choi ${ }^{1^{\dagger}}$, Seo-Yun Lee ${ }^{2}$ \\ ${ }^{1}$ Department of Health and Environment, Catholic Kwandong University, Gangneung 25601, Republic of Korea \\ ${ }^{2}$ Department of Physics, Kangwon National University, Chuncheon 24341, Republic of Korea
}

\begin{abstract}
Mass cultivation of microalgae is necessary to achieve economically feasible production of microalgal biodiesel. However, the high cost of nutrients is a major limitation. In this study, corncob extract (CCE) was used as an inorganic and organic nutrient source for the mass cultivation of Chlorella vulgaris (C. vulgaris). Chemical composition analysis of CCE revealed that it contained sufficient nutrients for mixotrophic cultivation of C. vulgaris. The highest specific grow rate of $\mathrm{C}$. vulgaris was obtained at $\mathrm{pH}$ of $7-8$, temperature of $25-30^{\circ} \mathrm{C}$, and CCE amount of $5 \mathrm{~g} / \mathrm{L}$. In the analysis using various growth models, Luong model was found to be the most suitable empirical formula for mass cultivation of $C$. vulgaris using CCE. Analysis of biomass and production of triacyglycerol showed that microalgae grown in CCE medium produced more than $17.23 \%$ and $3 \%$ more unsaturated fatty acids than cells cultured in Jaworski's Medium. These results suggest that growing microalgae in CCE-supplemented medium can increase lipid production. Therefore, CCE, agricultural byproduct, has potential use for mass cultivation of microalgae.
\end{abstract}

Keywords: Agricultural waste, Biomass, Carbon source, Corncob, Microalgae, Mixotrophic

\section{Introduction}

Discovering new alternative energy sources to replace petroleum-based fuels have become an important issue globally due to limited oil reserves and unstable oil prices. According to US Energy Information Administration, the supply of non-renewable energy sources such as oil is expected to reach peak output by 2020, after which crude oil production is expected to decline gradually [1]. Recently, biomass derived from microalgae is emerging as a new, sustainable, and clean energy resource for the production of third generation biofuels [2]. Choi and Lee [3] have reported that microalgae are suitable plants for the production of next-generation biodiesel with high biomass productivity and high lipid content. In addition, mass production of microalgae in combination with carbon sequestration and wastewater treatment has potential as green energy source from environmental point of view. Microalgae require less area than land-based plants. However, they can produce sustainable biomass with a faster growth rate [4]. In addition, microalgae are useful single-cell photo- synthetic organisms with many potential applications in biotechnology fields, including biofuels, pharmaceuticals, and aquaculture [3]. Despite these many advantages, the biggest obstacle to commercialization of microalgae-derived biodiesel is its production cost compared to fossil fuels. In order to lower the cost of biodiesel derived from microalgae, various factors need to be improved, including selection of microalgae strains, cultivation methods, nutrients, and carbon source. Among them, mass cultivation of microalgae using an inexpensive carbon source is the most important problem that needs to be solved first.

Microalgae are photosynthetic organisms with relatively simple growth requirements such as water, carbon dioxide, light, and nutrients. They have explosive growth potential under optimizing growth conditions [5]. Microalgae can cultivate under autotrophic, mixotrophic, and heterotrophic conditions. With external carbon source microalgae can produce 4-5 times more biomass than those under autotrophic conditions. In addition, lipid contents in microalgae cells are increased by over 40\% [6]. Xylose, saccharide, acetate, glucose, and methanol are generally used carbon sources for microalgae cultivation. Among them, the most commonly used

Received May 29, 2018 Accepted July 28, 2018

${ }^{\dagger}$ Corresponding author Email: hjchoi@cku.ac.kr Tel: +82-33-649-7297 Fax: +82-33-647-7635 ORCID: 0000-0003-3370-4277 
carbon source is glucose [7]. However, any external carbon source requires additional costs. The cost of such external carbon source is a hindrance to lowering the price of microalgae-derived biodiesel. To overcome this problem, this study was conducted to investigate the potential of using corncob extracts (CCE), an agricultural byproduct containing large amounts of glucose and minerals, as an external carbon source for microalgae cultivation. This study selected Chlorella vulgaris (C. vulgaris) among many microalgae. It has a high lipid content with resistance to environmental change. $C$. vulgaris has high protein content and balanced amino acid compositions. It has the potential to produce high value-added products useful for various fields such as pharmaceuticals, health foods, feeds, and nutrients [6].

Maize (Zea mays), one of the world's three major crops (along with rice and wheat), is used mainly as feed for livestock. It is also an important food resource in developing countries [8]. World corn production in 2016 was 1.114 billion tons, an increase of $1.1 \%$ compared to that in 2015 [9]. As the amount of corn produced is increased, the amount of corncob is also increased. Corncob is one of the most abundant agricultural wastes produced in the world [8]. Approximately $18 \mathrm{~kg}$ of corncobs is produced from $100 \mathrm{~kg}$ of corn grain [8, 9]. Previous mass balance analysis has revealed that $1,000 \mathrm{~g}$ of dried corncobs contain $139.8 \mathrm{~g}$ of xylooligosaccharides, $328.1 \mathrm{~g}$ of glucose, $25.1 \mathrm{~g}$ of cellobiose, and $147.8 \mathrm{~g}$ of xylose [10, 11]. Glucose and xylose are abundant in corncobs. Whether they can be used as an external carbon source for the growth of microalgae is currently unknown. Therefore, to objective of this study was to determine the effect of CCE on growth and lipid contents of $C$. vulgaris. For this propose, effects of $\mathrm{pH}$, temperature, and initial CCE concentration on microalgae cultivation were analyzed using Haldane, Double exponential, Edwards, Luong, Webb, Moser, and Teissier models $[12,13]$. Lipid contents of microalgae grown in CCE were also analyzed and compared to lipid contents of microalgae grown in other carbon sources.

\section{Materials and Methods}

\subsection{Materials}

Microalgae, C. vulgaris (KMCC-143), used in this experiment were purchased from Korea Ocean Research \& Development Institute (KAERI). They were grown in a thermostat at $25 \pm 1^{\circ} \mathrm{C}$ for 5 d using Jaworski's Medium (JM). C. vulgaris was cultivated with LEDs of white color, $120 \mu \mathrm{mol} / \mathrm{m}^{2} \mathrm{~S}$ of light intensity, 16L: $8 \mathrm{D}$ of light period, and $\mathrm{pH}$ of 7 . The initial concentration of $C$. vulgaris was $0.357 \pm 0.7 \mathrm{~g} / \mathrm{L}$.

Corncobs were collected from farmhouses in Gangneung, Korea. To remove organic substances and pollutant on the surface of corncobs, they were washed several times with deionized water and dried in an oven at $80^{\circ} \mathrm{C}$ for $48 \mathrm{~h}$. The process of drying was to increase concentrations of organic and inorganic substances in corncobs, thereby increasing contents of organic and inorganic substances in CCE. These dried corncobs were pulverized into fine powder using a blender. Corncob powder of less than 80 mesh size was used. To obtain CCE, $50 \mathrm{~g}$ of dried corncob powder was added into $1,000 \mathrm{~mL}$ of $60 \%$ ethanol and shaken in a shake incubator (MR-R505, Mrga Science, Korea) at $60^{\circ} \mathrm{C}$ for $48 \mathrm{~h}$. CCE was then filtered with a membrane filter $(0.45 \mu \mathrm{m}$, Whatman) to separate residues. Ethanol was then evaporated at $70^{\circ} \mathrm{C}$ for 5 h using a rotary evaporator (EYELA N-1000, Rikakkai Co., Ltd., Japan). After ethanol was removed, CCE was refrigerated at $4^{\circ} \mathrm{C}$ or lower. CCE was then diluted to designated concentration according to experimental plan and used for microalgae growth experiment.

\subsection{Experimental Design}

Batch-experiments were carried out. Temperature, photoperiod, and light intensity were kept the same as culture conditions. In this experiment JM medium was used to compare growth rate of biomass and lipid content in CCE. JM medium is standard media for cultivation of $C$. vulgaris. In a $10 \mathrm{~L}$ reactor, $5 \mathrm{~L}$ of $\mathrm{JM}$, microalgae, and various amounts (0-25 g/L) of CCE were mixed according to the experimental design. The mixed solution was reacted for $5 \mathrm{~d}$ with stirring at $150 \mathrm{rpm}$. Growth rate of $C$. vulgaris was measured by sampling ( $10 \mathrm{~mL})$ at a fixed time every day. The $\mathrm{pH}$ was controlled from 3 to 10 with $0.5 \mathrm{~mol} \mathrm{NaOH}$ and/or $\mathrm{HCl}$. Temperature was adjusted to $15-40^{\circ} \mathrm{C}$. All experiments were carried out with fixed parameters for the measurement of one parameter.

\subsection{Method for Analysis}

Qualitative and quantitative analyses of inorganic components contained in corncob were performed using X-ray diffraction (XRD) (XRF-1500, Shimadzu, Japan). Components of CCE were analyzed by liquid chromatography (Agilent 1290, Agilent Technol., USA). Growth rate $(\mu)$ of $C$. vulgaris was calculated as follows.

$$
\mu=\frac{1 n \frac{X_{1}}{X_{0}}}{t-t_{0}}
$$

where $\mu$ was the growth rate (1/d), $X_{0}$ and $X_{1}$ were $C$. vulgaris concentrations $(\mathrm{g} / \mathrm{L})$ initially and at a constant cultivation time, respectively, while $t$ was cultivation time (day).

Dry mass of microalgae was measured by filtering $50 \mathrm{~mL}$ of sample with GF/C (Whatmann, UK) and drying at $105^{\circ} \mathrm{C}$ for 24 $\mathrm{h}$ before measuring its mass.

$$
C_{B}=\frac{C_{b}-C_{b 0}}{t-t_{0}}
$$

where $C_{B}$ was the amount of biomass, $C_{b}$ and $C_{b 0}$ were amounts of biomass at $t$ and $t_{0}$, respectively. Growth and inhibitory effects of $C$. vulgaris on various conditions were analyzed using Haldane, Double exponential, Edwards, Luong, Webb, Moser, and Teissier models. $\mathrm{pH}$ was measured with a $\mathrm{pH}$ meter (ISTEK, $\mathrm{pH}-20 \mathrm{~N})$.

Oil extraction analysis was performed by extracting wet oil using C. vulgaris harvested by centrifugation at $628 \mathrm{~g}$ for $15 \mathrm{~min}$. Pure nucleic acid (Junsei 96\%, Japan) was used as solvent. Wet 
oil extraction was carried out using $3.3 \mathrm{~mL}$ of samples containing $1 \mathrm{~g}$ of $C$. vulgaris. The amount of microalgae was $5 \%(\mathrm{w} / \mathrm{w})$ relative to solvent. After $C$. vulgaris mixed with nucleic acid was stirred for $3 \mathrm{~h}$, the solvent layer including oil was recovered and evaporated under reduced pressure (Genevac, EZ2 PLUS). After solvent was volatilized, the remaining oil was recovered. The above extraction procedure was repeated three times for complete recovery of oil. The amount of microalgae oil extracted was calculated as follows.

$$
\begin{aligned}
& \text { Amount of oil extraction }\left(\%, \frac{w}{w}\right)= \\
& \frac{\text { Amount of recovered oil }(\mathrm{g})}{\text { Amount of dried microalgae }(\mathrm{g})} \times 100
\end{aligned}
$$

A modified direct trans-esterification method was used to determine total fatty acid content of microalgae and extracted microalgae oil [3]. Fatty acid methyl esters (FAMEs) analysis of microalgae was carried out according to EN ISO 5508 and EN ISO 550 after analyzing fatty acid content (mg/g oil) of the extracted oil. All experiments were repeated five times or more. Average data were calculated and used.

\section{Results and Discussion}

\subsection{Characteristics of Corncob Extract}

Corncobs contain about $39.1 \%$ cellulose, $42.1 \%$ hemicellulose, 9.1\% lignin, $1.7 \%$ protein, and $1.2 \%$ ash [10]. Sugar is the most abundant in CCE. Moreover, CCE contains $1.84 \%$ of protein, $1.18 \%$ of fat, and $24.28 \%$ of various minerals such as $\mathrm{K}, \mathrm{P}$, $\mathrm{Ca}, \mathrm{Mg}$, and Fe $[11,14]$. In particular, glucose, xylose and arabinose had high amounts in CCE $(47.71 \%, 32.26 \%$, and $10.42 \%$, respectively) (Table 1). As an external carbon source for mass cultivation of microalgae, glucose or glycerol is mainly used because they can improve photosynthesis and absorption ability of organic matter of microalgae with relatively low cost [15]. Since glucoside component of CCE accounted for nearly half of its total sugar content, CCE might be a good source for microalgae growth.

Table 1. Sugar Compositions of Corncob Extract

\begin{tabular}{clc}
\hline & Composition & Corncob extract \\
\hline & Glucose & 47.71 \\
\cline { 2 - 3 } Xylose & 32.26 \\
\cline { 2 - 3 } Sugar & Arabinose & 10.42 \\
\cline { 2 - 3 } composition $[\%]$ & Sucrose & 4.14 \\
\cline { 2 - 3 } & Galatose & 3.28 \\
\cline { 2 - 3 } & Fluctose & 0.35 \\
\cline { 2 - 3 } & Arabinose/Xylose & 0.07 \\
\cline { 2 - 3 } & Lignin & 0.34 \\
\cline { 2 - 3 } & Other & 0.07 \\
\hline
\end{tabular}

\subsection{Effect of Different Parameters}

\subsubsection{Effect of $\mathrm{pH}$}

The $\mathrm{pH}$ determines the solubility of $\mathrm{CO}_{2}$ and minerals in microalgae media. It directly or indirectly affects the metabolism of microalgae $[3,5]$. To investigate the effect of $\mathrm{pH}$ on mass culture of microalgae using CCE, the temperature was controlled at $25 \pm 0.3^{\circ} \mathrm{C}$, the concentration of CCE was fixed at $5 \mathrm{~g} / \mathrm{L}$, and the $\mathrm{pH}$ was adjusted to 3-12. C. vulgaris was cultivated for $5 \mathrm{~d}$ using various $\mathrm{pH}$ values. Its average growth rate was then calculated. Results are shown in Fig. 1. The growth rate of $C$. vulgaris was $0.305-0.3856\left[\mathrm{~d}^{-1}\right]$. It was the highest at $\mathrm{pH}$ 8. Its growth rate was greatly decreased with increasing $\mathrm{pH}$. In particular, the growth rate of $C$. vulgaris was found to be $0.129-0.183\left[\mathrm{~d}^{-1}\right]$ at $\mathrm{pH} 10,0.092-0.126\left[\mathrm{~d}^{-1}\right]$ at $\mathrm{pH} 11$, and 0.002-0.095 $\left[\mathrm{d}^{-1}\right]$ at $\mathrm{pH} 12$. This indicated that the growth of $C$. vulgaris was almost stopped at $\mathrm{pH} 10$. Growth rates of $C$. vulgaris at $\mathrm{pH} 7$ and $\mathrm{pH} 8$ in JM medium were found to be 0.09 and $0.11\left[\mathrm{~d}^{-1}\right]$, respectively. These results showed that the growth rate of $C$. vulgaris in the presence of CCE was significantly decreased with increasing $\mathrm{pH}$ above 10 .

It is known that $\mathrm{pH}$ is a major factor that affects the solubility of inorganic carbon (DTC) [6]. Most inorganic carbon is present as $\mathrm{CO}_{2}$ when $\mathrm{pH}$ is less than 5 . At $\mathrm{pH}=6.6$, both $\mathrm{CO}_{2}$ and $\mathrm{HCO}_{3}$ are present in the same amount. At pH 8.3, almost all inorganic carbon is present as $\mathrm{HCO}_{3}$. Therefore, $\mathrm{pH}$ should be controlled during cultivation to increase the absorption and availability of $\mathrm{CO}_{2}$ by microalgae [16]. The $\mathrm{pH}$ can also directly affect the permeability of microalgae cell and hydronium form of inorganic salt, thus indirectly affecting the absorption of inorganic salts $[6,17]$. During cultivation of microalgae, $\mathrm{CO}_{2}$ is consumed by microalgae due to photosynthesis, thus increasing the $\mathrm{pH}$ of medium. Therefore, it is necessary to add a substance such as hydrochloric acid or acetic acid to control the $\mathrm{pH}$ so that it will not become too high. Acetic acid is advantageous in that it can be used as a carbon source to improve microalgae growth rate in addition to $\mathrm{pH}$ control compared to hydrochloric acid. In general, microalgae can convert inorganic carbon in the form of $\mathrm{CO}_{2}$ and $\mathrm{HCO}_{3}$ into $\mathrm{CO}_{2}$ form through carbonic anhydrase and use it for photosynthesis and growth [18]. Therefore, the optimum $\mathrm{pH}$ for culturing $C$. vulgaris is $\mathrm{pH} 8$, which is the most abundant form of $\mathrm{HCO}_{3}$. Its optimum $\mathrm{pH}$ range is 7-9. In particular, inorganic carbon exists in the form of $\mathrm{CO}_{3}$ at $\mathrm{pH}$ above 10, making it difficult

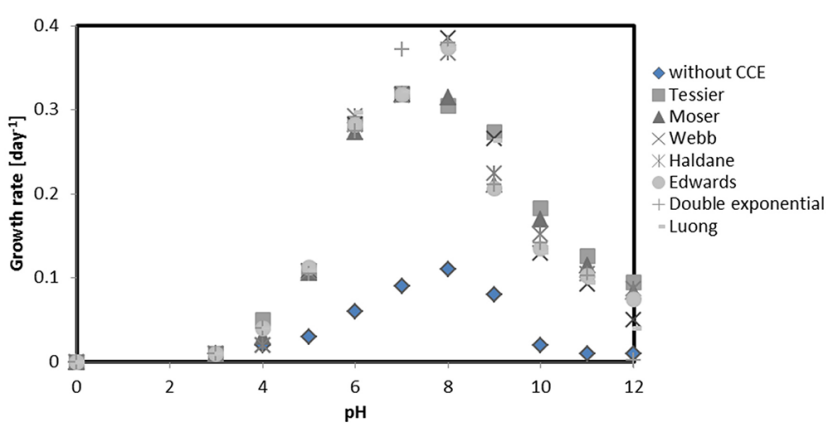

Fig. 1. Maximum specific grow rate of $\mathrm{C}$. vulgaris at various $\mathrm{pH}$ using various inhibition models (cultivation of $5 \mathrm{~d}$, CCE concentration of $5 \mathrm{~g} / \mathrm{L}$, and temperature of $25^{\circ} \mathrm{C}$ ). 
to be absorbed by $C$. vulgaris. Therefore, the growth of microalgae at $\mathrm{pH}$ above 10 is inhibited.

C. vulgaris generally shows the best growth rate at $\mathrm{pH} 7-8$, although there are some $\mathrm{pH}$ variations depending on species of microalgae [16, 19]. The maximum growth rate of $C$. vulgaris in the presence of CCE was 3.0-3.5 times higher than that without CCE. This indicated that carbon sources contained in CCE provided excellent external nutrients for $C$. vulgaris growth. As a result of this experiment, the growth rate of $C$. vulgaris was increased with increasing $\mathrm{pH}$ in all growth models. Significantly higher growth rate was started at $\mathrm{pH}$ above 6 .

\subsubsection{Effect of initial CCE concentration}

Various nutrients such as carbon, oxygen, nitrogen, potassium, calcium, magnesium, iron, sulfur, phosphorus (P), and trace elements are needed for the growth of microalgae [20]. The main nutrients are carbon, oxygen, nitrogen, phosphorus, and potassium. Carbon and oxygen can be obtained from water and air. However, nitrogen, phosphorus, and potassium must be absorbed from the medium [21]. During microalgae cultivation, N and $\mathrm{P}$ are very limited. However, both of which play important role in regulating the growth rate and lipid production of microalgae [22]. Glycerol and glucose are extensively used as external carbon sources for microalgae mass cultivation. However, previous studies have reported that if their concentration is too high, they can inhibit the growth of microalgae [23, 24]. Therefore, optimizing the concentration of external carbon sources for mass cultivation of microalgae is important in addition to $\mathrm{pH}$ and temperature. To determine the effect of CCE on microalgae growth, various concentrations (0-25 g/L) of CCE were used according to the results of previous experiments while the $\mathrm{pH}$ and temperature were fixed at 7 and $25^{\circ} \mathrm{C}$, respectively. Results indicated that the growth rate of $C$. vulgaris was increased with increasing concentration of CCE up to $5 \mathrm{~g} / \mathrm{L} \mathrm{CCE}$ in all growth models (Fig. 2). The highest growth rate of $C$. vulgaris was obtained at $0.3856\left[\mathrm{~d}^{-1}\right]$ in Webb model, followed by those in Double exponential, Edwards, and Luong models $\left(0.379,0.374\right.$, and $0.373\left[\mathrm{~d}^{-1}\right]$, respectively). When CCE concentration was increased to be above $10 \mathrm{~g} / \mathrm{L}$, the growth rate of $C$. vulgaris was decreased. When CCE concentration was increased to $20 \mathrm{~g} / \mathrm{L}$, the growth rate of $C$. vulgaris was decreased to $0.141-0.175\left[\mathrm{~d}^{-1}\right]$. Moreover, the color of $C$. vulgaris was slowly changed from green to brown after $4 \mathrm{~d}$ of cultivation at CCE concentration above $15 \mathrm{~g} / \mathrm{L}$. This suggests that the medium might have been contaminated by microorganisms from the atmosphere due to excess nutrients remaining in the medium and microalgae growth inhibition by the excessive nutrient content of CCE. Liang et al. [21] have also reported that the growth rate of microalgae is increased when glucose concentration is below $15 \mathrm{~g} / \mathrm{L}$. However, glucose concentration over $15 \mathrm{~g} / \mathrm{L}$ inhibited the growth of microalgae. In addition, too much nutrient caused massive growth of fungi in the medium [21]. Our results also confirmed that white fungi grew when CCE concentration was at $15 \mathrm{~g} / \mathrm{L}$. These white fungi grew more actively as the concentration of CCE was increased. A large amount of glucoside $\left(\mathrm{C}_{6} \mathrm{H}_{12} \mathrm{O}_{6}\right)$ is present in CCE. As a major compound of carbohydrate metabolism, glucoside is a type of aldehyde group-bearing sugar. It is often present in hexagonal rather than chainlike form [3]. Glucose has a high

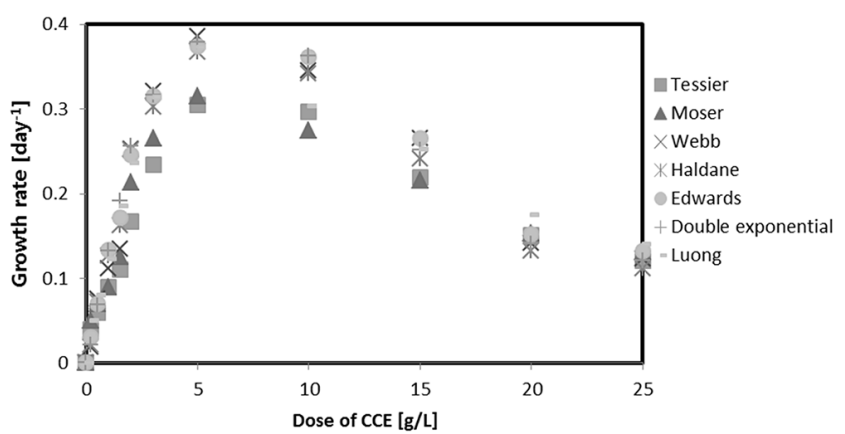

Fig. 2. Maximum specific grow rate of $C$. vulgaris at different concentrations of CCE ( $\mathrm{pH}$ of 7 , temperature of $25^{\circ} \mathrm{C}$, and cultivation period of $5 \mathrm{~d}$ ).

energy content per mol compared to other substrates such as acetate, indicating that it might provide high growth rate and respiration rate for microalgae [25]. Accordingly, a large amount of glucose in the medium would not only promote growth of microalgae, but also propagate white fungi which can inhibit the growth of microalgae. Therefore, $5 \mathrm{~g} / \mathrm{L}$ CCE is recommended for mass cultivation of $C$. vulgaris to prevent fungal growth.

\subsubsection{Effect of temperature}

Temperature is an important parameter affecting the growth of microalgae such as CCE concentration and $\mathrm{pH}$. This is because temperature affects growth rate, cell size, biochemical components, and nutrient requirements in microalgae growth [16]. To determine the effect of temperature on the growth of $C$. vulgaris in this study, the optimum temperature condition was determined by fixing the concentration of CCE at $5 \mathrm{~g} / \mathrm{L}, \mathrm{pH}$ at 7-8, and cultivation time at $7 \mathrm{~d}$. Experimental results showed that the best temperature for growing C. vulgaris was between 25 and $30^{\circ} \mathrm{C}$. The growth rate of $C$. vulgaris was decreased at temperatures above $30^{\circ} \mathrm{C}$ or below $20^{\circ} \mathrm{C}$ (Fig. 3). The optimal temperature for microalgae such as Chlorella and Scenedesmus in general is $25-30^{\circ} \mathrm{C}$ [7]. The same optimal temperature was found for $C$. vulgaris in the presence of CCE. Since microalgae are not capable of regulating temperature inside cells, optimum temperature should be maintained for their mass culture [4, 23]. In general,

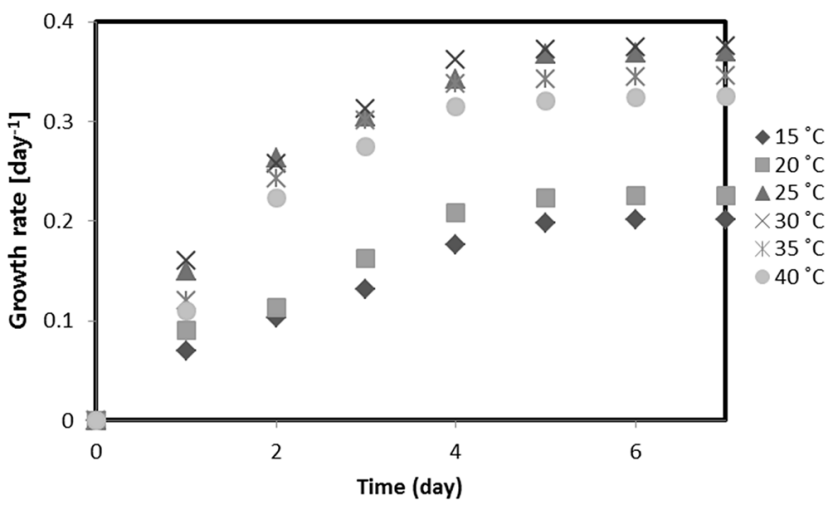

Fig. 3. Specific grow rate of $C$. vulgaris at different temperatures $(\mathrm{pH}$ of 7-8, CCE concentration of $5 \mathrm{~g} / \mathrm{L}$, and cultivation period of $7 \mathrm{~d}$ ). 
microalgae are cultivated at temperature between 18 and $35^{\circ} \mathrm{C}$ depending on medium composition, cultivation conditions, and microalgae species. When temperature is too low (less than $16^{\circ} \mathrm{C}$ ), the growth rate of microalgae will slow down. When the temperature is too high (more than $35^{\circ} \mathrm{C}$ ), enzyme activity in microalgae will be inhibited and their photosynthesis activity will be decreased [26].

\subsection{Effect of CCE on Biomass of $C$. vulgaris}

The effect of CCE on the biomass of $C$. vulgaris was investigated using the above parameter optimization experiment at $\mathrm{pH}$ of 7-8, temperature of $25^{\circ} \mathrm{C}$, and CCE concentration of $5 \mathrm{~g} / \mathrm{L}$. Experimental results are shown in Fig. 4 . The biomass of $C$. vulgaris in the presence of CCE was increased continuously until $5 \mathrm{~d}$, reaching $3.12 \mathrm{~g} / \mathrm{L}$ of biomass. There was no significant change thereafter. These microalgae cultivated in JM without CCE were found to have biomass of $0.792 \mathrm{~g} / \mathrm{L}$ after $5 \mathrm{~d}$ of growth. Therefore, the biomass of $C$. vulgaris in the presence of CCE was about four times higher than that cultured in JM. Since corncob is an abandoned agricultural by-product, it is very inexpensive and environmentally friendly. Using it for culturing $C$. vulgaris will be significant in terms of resource recycling.

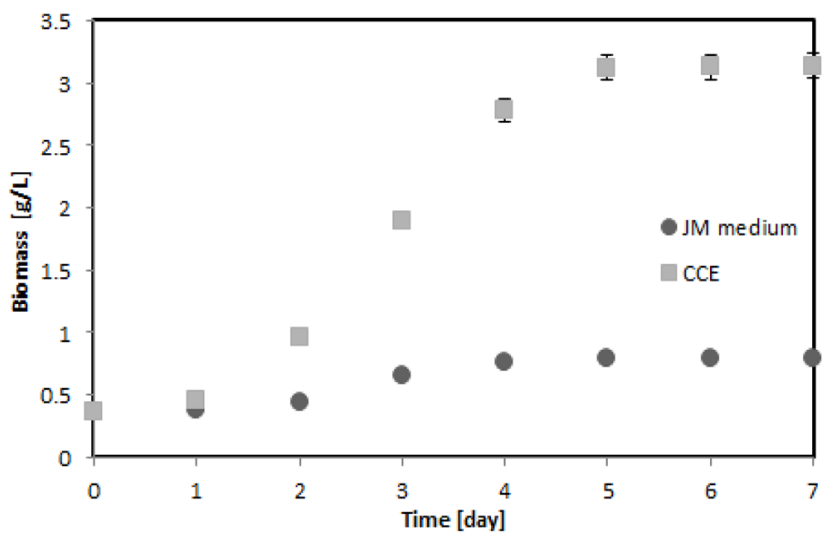

Fig. 4. Effect of corncob extract on biomass of $\mathrm{C}$. vulgaris ( $\mathrm{pH}$ of 7-8, CCE concentration of $5 \mathrm{~g} / \mathrm{L}$, and temperature of $25^{\circ} \mathrm{C}$ ).
In this study, the effect of CCE on growth of $C$. vulgaris was determined using various models such as Moser, Tessier, Webb, Haldane, Edwards, Double exponential, and Luong model. Each of these models is a modified Mond equation. Parameters are upgraded by empirical equations according to various conditions to modify and supplement the Mond model [12]. The Luong model describes substrate inhibition kinetics of cell growth with a single restriction substrate. The Haldane equation is widely used to explain substrate inhibition dynamics and biodegradation of inhibitory substrates. Moser upgraded Monod's model as a typical parameter to incorporate effects of microbial adoption into a fixed process by mutation [13]. In this study, experimental results were compared and analyzed using various control models to find suitable parameters for mass cultivation of $C$. vulgaris and obtain reliable data through comparative analysis. The maximum growth rate according to the above optimization experiment was calculated using various control models. Results are summarized in Table 2. The maximum growth rate was from 0.305 to $0.386\left[\mathrm{~d}^{-1}\right]$. The relationship $\left(\mathrm{R}^{2}\right)$ of the Luong model was the highest (at 0.9916). Therefore, the Luong model was the most suitable one for $C$. vulgaris growth experiments using CCE.

Results of comparison to previous studies using various external carbon sources for mass cultivation of $C$. vulgaris are shown in Table 3. Cultivation of $C$. vulgaris can use relatively cheap glycerol or glucose as an external carbon source. Recently, dairy wastewater, wine residue, or wastewater from tofu factory has also been used as external carbon source [4, 27]. Although glycerol and glucose are relatively inexpensive external carbon sources, they can be costly to cultivate microalgae in large quantities. In the case of using dairy wastewater, wine residue, or wastewater from tofu factories, it has disadvantage because environmental conditions such as $\mathrm{pH}$, salinity, nutrient concentration, and temperature suitable for microalgae growth need to be controlled $[16,19]$. When microalgae are cultivated using the CCE, there is no need to specially control these parameters. The application is very simple and the waste is recycled, making it very economical. Compared to previous studies, the yield of biomass using CCE was 0.83 to 3 times higher than that with other carbon sources.

Table 2. Estimated Parameters of Various Substrate Inhibition Models

\begin{tabular}{|c|c|c|c|}
\hline Model & Equation & Parameters & $\mathbf{R}^{2}$ \\
\hline Moser & $\mu=\mu_{m} S^{n} /\left(K_{s}+S^{n}\right)$ & $n=0.6732, K_{s}=0.8245, \mu_{m}=0.315$ & 0.9613 \\
\hline Tessier & $\mu=\mu_{m}\left[1-\exp \left(-S / K_{s}\right)\right]$ & $K_{s}=0.5425, \mu_{m}=0.305$ & 0.9245 \\
\hline Webb & $\mu=\left\{\mu_{m} S\left[1+\left(S / K_{i}\right)\right]\right\} /\left\{K_{s}+S+\left(S^{2} / K_{i}\right)\right\}$ & $K_{i}=1.168, K_{s}=0.3315, \mu_{m}=0.386$ & 0.9357 \\
\hline Haldane & $\mu=\mu_{m} S /\left[\left(K_{s}+S\right)+\left(1+S / K_{i}\right)\right]$ & $K_{i}=1.131, K_{s}=1.123, \mu_{m}=0.367$ & 0.8997 \\
\hline Edwards & $\mu=\left[\mu_{m} S /\left(K_{s}+S\right)\right] \exp \left(-S / K_{i}\right)$ & $K_{i}=4.314, K_{s}=0.8173, \mu_{m}=0.374$ & 0.9586 \\
\hline Double exponential & $\mu=\mu_{m}\left[\exp \left(-S / K_{i}\right)-\exp \left(-S / K_{s}\right)\right]$ & $K_{i}=4.576, K_{s}=0.24, \mu_{m}=0.379$ & 0.9542 \\
\hline Luong & $\mu=\mu_{m} S /\left(K_{s}+S\right)\left(1-S / S_{m}\right) \mathrm{n}$ & $S_{m}=19.84, K_{s}=0.7108, \mu_{m}=0.381, n=2.657$ & 0.9916 \\
\hline
\end{tabular}

where $\mu$ and $\mu_{m}$ are the specific growth rate and maximum specific growth rate, respectively. $S$, $K_{s}$, and $S_{m}$ are limiting substrate concentration (corncob extracts in this study), the Monod half saturation constant, and the maximum substrate inhibitory concentration at which no growth was observed, respectively. $n$ is the constant that accounts the relationship between $\mu$ and $S$. 
Table 3. Comparison of Mixotrophic Performance of C. vulgaris Cultivated under Different Conditions According to Literature and in This Work

\begin{tabular}{|c|c|c|c|c|c|}
\hline Microalgal strain & Carbon source & Carbon amount $[\mathrm{g} / \mathrm{L}]$ & Culture conditions & Biomass [g/L] & Reference \\
\hline Chlorella vulgaris & Cheese whey & 2.2 (hydrolyzed) & Continuos & 2.59 & [28] \\
\hline Chlorella vulgaris & Glycerol + UF & 2.2 & Continuos & 1.67 & [28] \\
\hline Chlorella vulgaris & White wine less & 2.2 & Continuos & 1.75 & [28] \\
\hline Chlorella vulgaris & Glucose & 4 & Batch flasks & 1.4 & [7] \\
\hline Chlorella vulgaris & Glucose + Galactose & 10 & Batch & 2.24 & [25] \\
\hline Chlorella vulgaris & Glycerol + glucose & $5+2$ & Batch & 2.6 & {$[25]$} \\
\hline Chlorella vulgaris & Acetate & 10 & Batch & 0.99 & {$[21]$} \\
\hline Chlorella vulgaris & Corncob extract & 5 & Batch & 3.13 & This study \\
\hline
\end{tabular}

\subsection{Concentrations and Compositions of Fatty Acid Methyl Esters (FAMEs)}

Microalgae contain large amounts of triacylglycerols (TAGs) (30\% to $60 \%$ of dry weight) through spontaneous accumulation depending on their species. Their photosynthetic efficiency and lipid production potential are also higher than land crops [29]. TAGs are major sources of biofuels. They are neutral compounds stored predominantly in microalgae under stressful conditions such as strong and abundant light or malnutrition. That is, under environmental stress conditions such as nutrient starvation and intense light, microalgae can rapidly stop dividing. TAGs are accumulated as primary compounds. Under these stress conditions, synthesized fatty acids are mainly used to accumulate TAGs. If stress conditions continue, intracellular proteins are also used for the synthesis of TAGs $[23,30]$.

Glucose in CCE is decomposed through cell respiration of microalgae to produce energy. The energy is stored in the form of adenosine triphosphate (ATP). This energy is used for the breathing of microalgae [14]. Glucose biosynthesis of microalgae is synthesized by trios of energy, carbon dioxide, and water. It is produced by photosynthesis reaction. Based on this synthesis, hexosuccine is synthesized and stored as starch in microalgae cells $[25,26]$. These stored starches are then converted to triglycerides. These triglycerides can be used as raw materials for biodiesel. Therefore, when glucose of CCE is used as an organic carbon source, the amount of stored fat is increased and lipid content is also increased.

Results of comparison of total TAGs of $C$. vulgaris cultivated in CCE and JM are shown in Table 4. TAGs contents in $C$. vulgaris cultivated in CCE and JM were $14.71 \%$ and $31.94 \%$, respectively. C. vulgaris grown in CCE had $17.23 \%$ more TAGs than that cultivated in JM. This suggests that CCE contains a lot of glucose and other sugar components, which might have affected the accumulation of TAGs content in $C$. vulgaris.

The detailed mechanism involved in the synthesis of fatty acids and TAGs in microalgae is not known yet. However, biodiesel can be produced by converting TAGs contained in microalgae through trans-esterification. Therefore, the higher the TAGs content, the more production of biodiesel [7, 23]. Lipid content of microalgae varies depending on culture conditions. In general, the lipid content of $C$. vulgaris is around $14.5 \%$ under authotrophic conditions. It is increased to $20 \%$ and $30 \%$ under heterotrophic and mixotrophic conditions, respectively [21]. In particular, increasing glycerol amount in medium can improve photosynthesis and absorption capacity of microalgae. It also increases the lipid content in microalgae by $35 \%$ [23].

Microalgae are mostly single cells. Depending on species, chain length of fatty acids contained in microalgae varies from C10 to C24 [23]. Microalgae produce more oil per unit area than land-based crops. They can grow more than 20 times a year because

Table 4. Total TAG Content in Dry Mass of Investigated Algae Species and Compositions of Total Fatty Acid Profiles of C. vulgaris Oil

\begin{tabular}{|c|c|c|c|c|c|c|c|c|}
\hline \multicolumn{9}{|c|}{ Total TAG content in dry biomass of algae (\%) } \\
\hline \multicolumn{2}{|c|}{ JM medium } & \multicolumn{7}{|c|}{$14.71 \pm 0.51$} \\
\hline \multicolumn{2}{|c|}{ CCE } & \multicolumn{7}{|c|}{$31.94 \pm 1.21$} \\
\hline \multicolumn{9}{|c|}{ Composition of total fatty acids (\%) } \\
\hline Saturated & C14 & C16 & C17 & C18 & C20 & C22 & C24 & Total \\
\hline JM medium & $0.31 \pm 0.01$ & $30.11 \pm 0.52$ & $1.45 \pm 0.03$ & $4.15 \pm 0.11$ & $0.58 \pm 0.02$ & $0.25 \pm 0.03$ & $0.13 \pm 0.01$ & $36.98 \pm 1.23$ \\
\hline CCE & $0.39 \pm 0.02$ & $22.54 \pm 0.43$ & $1.71 \pm 0.03$ & $7.56 \pm 0.05$ & $0.13 \pm 0.14$ & $1.27 \pm 0.11$ & $0.22 \pm 0.03$ & $33.82 \pm 1.18$ \\
\hline Unsaturated & C16:1 & C18:1 & C18:2 & C18:3 & C20:1 & C22:1 & C24:1 & Total \\
\hline JM medium & $4.23 \pm 0.12$ & $41.01 \pm 1.42$ & $10.91 \pm 1.13$ & $6.11 \pm 1.08$ & $0.53 \pm 0.02$ & $0.21 \pm 0.01$ & $0.02 \pm 0.01$ & $63.02 \pm 2.47$ \\
\hline CCE & $3.11 \pm 0.03$ & $43.19 \pm 2.15$ & $11.57 \pm 1.08$ & $7.31 \pm 1.41$ & $0.43 \pm 0.05$ & $0.52 \pm 0.07$ & $0.05 \pm 0.03$ & $66.18 \pm 2.63$ \\
\hline
\end{tabular}

Data were from 7-day cell growth at $\mathrm{pH} 7-8$, CCE concentration of $5 \mathrm{~g} / \mathrm{L}$, and temperature of $25^{\circ} \mathrm{C}$.

TAG: Triacylglycerols; CCE: Corncob Extracts. 
of its rapid growth rate [23]. In order to use vegetable oil and animal oil as biodiesel, the content of saturated fatty acid is important. High content of saturated fatty acids means that biodiesel has high degree of saturation. It can easily cause problems when using biodiesel higher than gasoline in winter due to its hardness [7, 16]. In addition, when a large amount of saturated fatty acid is contained, many alcohols are required in ester reaction in order to produce biodiesel. If ester reaction is insufficient, the viscosity will increase greatly and the piping will become clogged [16]. Therefore, the content of saturated fatty acids is important when using of vegetable oil or animal oil as biodiesel.

Major contents of $C$. vulgaris cultivated in the presence or absence of CCE were C16 or C18 saturated fatty acids and C18:1 or C18:2 unsaturated fatty acids. However, total saturated fatty acid content was $36.98 \%$ in the presence of CCE and $33.82 \%$ in the absence of CCE (Table 4). Therefore, C. vulgaris grown in CCE showed a decrease of $3 \%$ in saturated fatty acid content but an increase of $3 \%$ in unsaturated fatty acid content than C. vulgaris grown in JM. The higher content of unsaturated fatty acids in biodiesel component, the faster the automatic oxidation processes. Especially, contents of C18:1, C18:2, and C18:3 in $C$. vulgaris grown in CCE were higher than those of microalgae grown in JM. C18:0 stearic in saturated fatty acid is stable. C18:2 linoleic oxidation is faster than C18:1 oleic, while C18:3 linolenic oxidation is faster than C18:2 linoleic. As the level of unsaturation is increased, the oxidation is about 10 times faster [4, 23]. That is, C18:3 can oxidize 100 times faster than C18:1. Purity is very important for biodiesel [26]. Generally, the low level of C16:1 and C18:1 decreases the oxidative stability and cold flow [31]. Compared to rapeseed oil, algal oil from this study is not as rich in polyunsaturated fatty acids. Furthermore, in our study, the content of linolenic acid (18:3) in the three algae species was shown to correspond to the requirements of the EN 14103 standard, which states that the content of linolenic acid methyl ester in biodiesel fuel should not exceed $12 \%$. Thus, the algae strains used in this study could be considered promising, since the biodiesel fuel produced from them may potentially meet the requirements for the linolenic acid methyl ester content.

Various carbon sources such as sodium acetate [7, 21], fructose $[15,26]$, glucose [3, 25], glycerol [22, 24, 25], sucrose [15], and acetate [7] have been successfully used to increase growth rate and lipid content of microalgae. However, these methods require additional cost. The prices of glucose, glycerol, and acetate are currently 0.5-0.8, 0.6-0.7, and 0.9-0.94 US dollars per kg, respectively [26, 27]. Therefore, carbon source of CCE used in this study is very useful in terms of resource circulation because it recycles waste. In addition, the process is simple, inexpensive, and very effective for increasing microalgae growth rate and lipid content.

\section{Conclusions}

In order to investigate the effect of CCE on the growth of $C$. vulgaris, growth rate of microalgae was investigated using various growth control models based on $\mathrm{pH}$, temperature and the amount of CCE. In addition, the biomass was harvested and contents of TAGs and fatty acids of $C$. vulgaris cultivated in the presence or absence of CCE were compared and analyzed. As a result, C. vulgaris cultivated at $\mathrm{pH}$ of $7-8$, temperature of $25-30^{\circ} \mathrm{C}$, and CCE concentration of $5 \mathrm{~g} / \mathrm{L}$ had the highest growth rate. More than 4 times of biomass could be harvested when CCE was used as an external carbon source. In the analysis using various growth models, Luong model was found to be the most suitable empirical formula for mass cultivation of $C$. vulgaris using CCE because it had the highest correlation coefficient. The content of TAGs in $C$. vulgaris cultivated in CCE was about $35 \%$ higher than that cultivated in JM. Compared to rapeseed oil, algal oil from this study is not as rich in polyunsaturated fatty acids. Furthermore, in our study, the content of linolenic acid (18:3) in the three algae species was shown to correspond to the requirements of the EN 14103 standard, which states that the content of linolenic acid methyl ester in biodiesel fuel should not exceed 12\%. These results suggest that CCE is very effective for increasing microalgae growth rate and lipid content.

\section{Acknowledgments}

This study was supported by a grant (2016005271) of the Basic Science Research Program through the National Research Foundation of Korea (NRF) funded by the Ministry of Education, Science and Technology, Republic of Korea.

\section{References}

1. EIA. Short-Term Energy Outlook (STEO), U.S. Energy Information Administration. 2017: 06.

2. Choi HJ. Effect of acorn powder on the biomass productivity of microalgae. J. Korean Soc. Water Environ. 2015;31:134-141.

3. Choi HJ, Lee JM. Application of saccharified acorn-starch for biomass and lipid accumulation of microalgae. J. Korean Soc. Water Environ. 2016;32:197-204.

4. Choi HJ. Dairy wastewater treatment using microalgae for potential biodiesel application. Environ. Eng. Res. 2016;21:393-400.

5. Abbreu AP, Fernandes B, Vicente AA, Teixeira J, Dragone G. Mixotropic cultivation of Chlorella vulgaris using industrial dairy waste as organic carbon source. Bioresour. Technol. 2012;118:61-66.

6. Gupta PL, Lee SM, Choi HJ. Integration of microalgae cultivation system for wastewater remediation and sustainable biomass production. World J. Microbiol. Biotechnol. 2016;32:1-11.

7. Heredia-Arroyo T, Wei W, Ruan R, Hu B. Mixotrophic cultivation of Chlorella vulgaris and its potential application for the oil accumulation from non-sugar materials. Biomass Bioenerg. 2044;35:2245-2253.

8. Choi HJ. Application of corncob for treatment of $\mathrm{Cu}(\mathrm{II})$ in aqueous solution. KSWST J. Water Treat. 2017;25:61-72.

9. FAO, FAO Cereal Supply and Demand Brief. 2016.

10. Li H, Dai Q, Ren J, et al. Effect of structural characteristics of corncob hemicelluloses fractionated by graded ethanol precipitation on furfural production. Carbohydr. Polym. 2016;136: 203-209. 
11. Zhao Y, Cao W, Wang Z, Zhang B, Chen K, Ouyang P. Enhanced succinic acid production from corncob hydrolysate by microbial electrolysis cells. Bioresour. Technol. 2016;202:152-157.

12. Choi HJ. Inhibition of the growth of harmful cyanobacterial blooms, Microcystis Aeruginosa using caffeine. KSWST J. Water Treat. 2016;24:111-122.

13. Choi HJ. Removal of Microcystis aeruginosa using pine needle extracts. J. Korean Soc. Water Environ. 2017;33:8-14.

14. Gowdhaman D, Ponnusami V. Production and optimization of xylooligosaccarides from corncob by Bacillus aerophilus KGJ2 xylanase and its antioxidant potential. Int. J. Biol. Macromol. 2015;79:595-600.

15. Mitra D, Leenwen JV, Lamsal B. Heterotrophic/mixotrophic cultivation of oleaginous Chlorella vulgaris on industrial co-products. Algal Res. 2012;1:40-48.

16. Junying ZHU, Junfeng RONG, Baoning ZONG. Factors in mass cultivation of microalgae for biodiesel. Chinese J. Catal. 2013;34:80-100.

17. Wang H, Zhang W, Chen L, Wang J, Liu T. The contamination and control of biological pollutants in mass cultivation of microalgae. Bioresour. Technol. 2013;128:745-750.

18. Ashokkumar V, Rengasamy R, Deepalakshmi S, Sivalingam A, Sivakumar P. Mass cultivation of microalgae and extraction of total hydrocarbons: A kinetic and thermodynamic study. Fuel 2014;119:308-312.

19. Jankowska E, Sahu AK, Oleskowicz-Popiel P. Biogas from microalgae: Review on microalgae's cultivation, harvesting and pretreatment for anaerobic digestion. Renew. Sust. Energ. Rev. 2017;75:692-709.

20. Gupta PL, Lee SM, Choi HJ. A mini review: Photobio-reactors for large scale algal cultivation. World J. Microbiol. Biotechnol. 2015;31:1409-1417.

21. Liang Y, Sarkany N, Cui Y. Biomass and lipid productivities of Chlorella vulgaris under autotrophic, heterotrophic and mix- otrophic growth conditions. Biotechnol. Lett. 2009;31:1043-1049,

22. Liang Y, Sarkany N, Cui Y, Blackburn JM. Batch stage study of lipid production from crude glycerol derived from yellow grease or animal fats through microalgal fermentation. Bioresour. Technol. 2010;101:6745-6750.

23. Choi HJ, Lee SM. Biomass and oil content of microalgae under mixotrophic conditions. Environ. Eng. Res. 2015;20:25-32.

24. Choi HJ, Yu SW. Influence of crude glycerol on the biomass and lipid content of microalgae. Biotechnol. Biotechnol. Equip. 2015;29:506-513.

25. Kong WB, Yang H, Cao YT, Song H, Hua SF, Xia CG. Effects of glycerol and glucose on the enhancement of biomass, lipid and soluble carbohydrate production by Chlorella vulgaris in mixotrophic cultures. Food Technol. Biotechnol. 2013;51:62-69.

26. Lin TS, Wu JY. Effect of carbon source on growth and lipid accumulation of newly isolated microalga cultured under mixotrophic condition. Bioresour. Technol. 2015;184:100-107.

27. Park WK, Moon MH, Kwak MS, et al. Use of orange peel extract for mixotrophic cultivation of Chlorella vulgaris: Increased production of biomass and FAMEs. Bioresour. Technol. 2014;171:343-349.

28. Salati S, D'Imporzano G, Menin B, et al. Mixptrophic cultivation of Chlorella for local protein production using agro-food by-products. Bioresour. Technol. 2017;230:82-89.

29. Yeh KL, Chang JS. Effects of cultivation conditions and media composition on cell growth and lipid productivity of indigenous microalga Chlorella vulgaris ESP-31. Bioresour. Technol. 2012;105:120-127.

30. Cheah WY, Ling TC, Show PL, Juan JC, Chang JS, Lee DJ. Cultivation in wastewaters for energy: A microalgae platform. Appl. Energ. 2016;179:609-625.

31. Baldev E, Mubarakali D, Saravanakumar K, et al. Unveiling algal cultivation using raceway ponds for biodiesel production and its quality assessment. Renew. Energ. 2018;123:486-498. 